\title{
High-Order Coefficients of Second-Order ODEs in Relation to Pre-Factors for Complex Parameter
}

\author{
Fatih Say $^{1}$ \\ ${ }^{1}$ Department of Mathematics, Faculty of Arts and Sciences, Ordu University, Ordu, Turkey
}

\author{
Article Info \\ Keywords: Asymptotic expansions, Ap- \\ proximation, Asymptotic beyond all or- \\ ders, Perturbation, Series solutions \\ 2010 AMS: 34E20, 34M35, 34M40, \\ $34 M 60$ \\ Received: 23 April 2020 \\ Accepted: 3 June 2020 \\ Available online: 10 June 2020
}

\begin{abstract}
In this study, we asymptotically reconsider the relations between the pre-factors of a general inhomogeneous second-order ordinary differential equation and the high-order coefficients of its asymptotic power series for complex values of the asymptotic parameter $\varepsilon_{1}$. The study provides a general formula for its generic high-order coefficients with the associated pre-factors for complex $\varepsilon_{1}$ based on the use of a well-known factorial divided by a power approach.
\end{abstract}

\section{Introduction}

Many of the essential properties of the ordinary differential equations (ODE) can be investigated by using asymptotic expansion methods such as perturbation methods of Poincaré [1, 2], method of matched asymptotic expansion [3], WKB approximation method [4, 5] and SCEM method [6]. The generic feature of the singular differential equations is that the high-order coefficients of the singular perturbation expansions always behave in the characteristic factorial divided by a power (factorial/power) form, and they factorially diverge for a wide range of singular perturbation problems. It is principally first discussed in detail by Dingle [7] and Berry [8]. In the companion paper [9], we already considered the link between the pre-factor functions of a particular type of second-order inhomogeneous ODEs and the associated high-order coefficients of the asymptotic expansion. Motivated by the previous study, in this paper, we will reapply the same idea permitted us to obtain the formulae in [9] to the asymptotic solution of the general differential equation in the case of small parameter $\varepsilon_{1}$ is complex-valued. We will address what difference it will make in the derived formulae. Once it is done, one could use them while addressing the asymptotic properties of the differential equations such as superasymptotics, hyperasymptotics and Stokes rays [8], [10]-[12] since the exponential asymptotics is usually discussed in the complex plane. For instance, Stokes rays are the local properties of the differential equations and across which the exponentially growing terms occur along the complex plane. For this reason alone, it is nice to interpret the findings of [9] in terms of the complex values of a small parameter. Moreover, the neglected highest derivative of the singular differential equations at leading order becomes important as it varies rapidly. Therefore, the asymptotic behavior of the differential equations (and integrals) has been comprehensively studied in detail in the last few decades and, as a consequence, the subject of exponential asymptotics is introduced, see for example [13]-[17] and references therein. For this reason, studying the asymptotic behavior of such equations, especially for the ones whose exact solutions cannot be derived via conventional asymptotic techniques, are always of great interest.

In this paper, albeit briefly, we reconsider whether the formulas in [9] can be further extended for complex values of the 
small parameter while addressing the general representations of the general inhomogeneous second-order ODE. We again take into account the factorial/power ansatz of high-order coefficients $[7,15,18]$ to capture the formulas in the derivation of the asymptotic expansions for the particular case of this paper. We present that the links between the pre-factors and the coefficients of the asymptotic expansion of the ODE work for the complex values of $\varepsilon_{1}$. The outline of the article is as follows: First, we introduce the illustrative singular ordinary differential equation and re-define the small parameter of the ODE in terms of its complex values in Section 2. We next expand the equation in the traditional asymptotic expansion method where we derive the leading order solution along with the recurrence relationship of the successive terms of the expansion. To be able to address and interpret the general form of the high-order coefficients in terms of the pre-factor functions of the ODE for the complex parameter, we employ the common and powerful factorial/power formula whereby we determine their relationships in the limit $n \rightarrow \infty$ in Section 3. We finish the study with the concluding remarks in Section 4 .

\section{The asymptotic expansion of ODE}

In order to be able to capture the relationship between the high-order terms of the expansion and the pre-factor functions, we will address the asymptotic expansion of the following singular inhomogeneous ODE of [9], that is,

$$
\varepsilon_{1} \frac{d^{2} w(z)}{d z^{2}}+\varepsilon_{1} f(z) \frac{d w(z)}{d z}+g(z) w(z)=t(z),
$$

in which $\varepsilon_{1} \in \mathbb{C}$ is the small perturbation parameter and pre-factor functions $f(z), g(z)$ and $t(z)$ are not constant. Before starting to study this section, let us first discuss the form of the asymptotic expansions occurring in exponential asymptotics. Divergent solutions of the differential equations including this particular case mostly appear in the following nature in exponential asymptotics [19]

$$
\begin{aligned}
w(z)=\left(w_{0}(z)+\varepsilon_{1} w_{1}(z)+\varepsilon_{1}^{2} w_{2}(z)\right. & \left.+\cdots+\varepsilon_{1}^{n} \frac{\Gamma(2 n+\beta)}{\chi_{1}(z)^{2 n+\beta}}\left(\sum_{k=0}^{\infty} \frac{A_{k}(z)}{(2 n+\beta)^{k}}\right)\right) \\
& +\left(\sum_{l=0}^{m-1} \varepsilon_{1}^{l} B_{l}(z)\right) \exp \left(-\frac{\chi_{1}(z)}{\varepsilon_{1}}\right)+\left(\sum_{t=0}^{s-1} \varepsilon_{1}^{t} C_{t}(z)\right) \exp \left(-\frac{\chi_{2}(z)}{\varepsilon_{1}}\right)+\mathrm{R}\left(\varepsilon_{1}, z\right)
\end{aligned}
$$

in which $\chi_{i \geq 1}(z)$ s are subject to every single singularity of the early ordered terms of each level, and $\mathrm{R}\left(\varepsilon_{1}, z\right)$ is the resultant remainder of the expansion with respect to the order of the first neglected term. As it suffices for this particular case, only $\chi_{1}(z), A_{k}(z)$ and $\beta$ will be addressed in (3.1) of the following section. Functions $\chi_{i \geq 2}(z), B_{l}(z)$ and $C_{t}(z)$ can be addressed, in a similar way, when needed. It is indeed one of the main ideas lying behind the exponential asymptotics, see [19]. This will particularly be discussed in the succeeding section. The reason that such expansions diverge is in fact the singular point(s) of their early terms; most particularly, it is $w_{0}(z)$ in this general case. Moreover, the Stokes rays usually sprout from the singular point(s) of the early terms. The exponentially small terms which occur in the form of $\exp \left(-\chi_{i \geq 1}(z) / \varepsilon_{1}\right)$ appear and disappear across the active Stokes rays, and this can be observed when analytically continued in the Argand diagram; particularly, this jump occurs smoothly via error function. Based on the sectors occurred by the Stokes rays in the diagram, associated sub-dominant exponential terms come into play. Thence, the subject of exponential asymptotics deals with this divergence and its relation with the exponentially small terms hidden behind algebraic order terms [20]. Furthermore, the magnitude of the powers of the exponentially small terms of (2.2) shows at which point the asymptotic expansions change their behavior from decreasing to diverging to infinity; for more details, see [19].

The equation (2.1) currently contains no complex parameter besides $\varepsilon_{1}$. Since we are only interested in finding the asymptotic solutions for $\varepsilon_{1}$ in terms of pre-factor functions $f(z), g(z)$ and $t(z)$, we need to first introduce complex $\varepsilon_{1}$ in a useful way. Unlike to [21] where the independent variable is changed by multiplying complex factor, we re-scale the small perturbation parameter in this case. In particular, to address whether the links between the factors and the expansion coefficients derived in [9] work for the complex values of $\varepsilon_{1}$ in the asymptotic procedure or not, we principally re-scale $\varepsilon_{1}$, without loss of generality, by

$$
\varepsilon_{1}=e^{i \theta} \varepsilon
$$

where $0<\varepsilon \ll 1$. To express herein that we will focus on the general form of the first summation of (2.2) in terms of pre-factor functions in our derivation since we are only concerned with the limits $n \rightarrow \infty$ and $\varepsilon \rightarrow 0$ for the singular ODEs in the form of this paper. Upon substitution of this re-scaled values of $\varepsilon_{1}$ into the original differential equation (2.1), we may find the following singular differential equation depending on $\theta$

$$
e^{i \theta} \varepsilon \frac{d^{2} w(z)}{d z^{2}}+e^{i \theta} \varepsilon f(z) \frac{d w(z)}{d z}+g(z) w(z)=t(z)
$$


The solution of ODEs by conventional asymptotic methods usually proceeds in a similar way, see [1, 2] for details. Therefore, we will first assume that a regular asymptotic expansion of the solution of equation (2.4) exists. We then substitute it into the equation and equate the factors of like powers of the small parameter on both sides. In particular, let us proceed with the usual approach that its asymptotic power series solution in powers of $\varepsilon$ is

$$
w(z) \sim \sum_{n=0}^{\infty} \varepsilon^{n} w_{n}(z)
$$

which is valid in the limit as $\varepsilon \rightarrow 0$. Because this series expansion of $w(z)$ must satisfy the differential equation (2.4), we employ the summation in the equation. After rearranging into a hierarchy of powers of $\varepsilon$, we find

$$
\sum_{n=1}^{\infty} \varepsilon^{n}\left[w_{n-1}^{\prime \prime}(z)+f(z) w_{n-1}^{\prime}(z)+e^{-i \theta} g(z) w_{n}(z)\right]+e^{-i \theta} g(z) w_{0}(z)=e^{-i \theta} t(z),
$$

where the prime ${ }^{\prime}$ indicates the differentiation of the functions respecting to $z$. Once the factors of like powers of $\varepsilon$ are equated for both sides of the asymptotic equality (2.6), the leading order solution $w_{0}(z)$ at $\mathrm{O}(1)$ and the differential recurrence relation of $w_{n-1}(z)$ and $w_{n}(z)$ of the expansion in (2.6) at $\mathrm{O}\left(\varepsilon^{n}\right)$ are derived, respectively, as follows

$$
\begin{gathered}
w_{0}(z)=\frac{t(z)}{g(z)}, \\
w_{n-1}^{\prime \prime}(z)+f(z) w_{n-1}^{\prime}(z)+e^{-i \theta} g(z) w_{n}(z)=0,
\end{gathered}
$$

for $n \geq 1$. An observant reader may notice that the low-ordered term $w_{0}(z)$ is not affected with the complex values of the small parameter and it is the same as the corresponding one of [9]; in fact, this reinforces the consistency between the two pieces of the works. When the leading order term and then the associated succeeding terms of the expansion are employed repeatedly in the above sequence (2.8), one can derive the high-order terms as $n$ increases in practice by earlier terms. However, one must make sure that singularity or singularities of the low-ordered term(s) must be secured in the high-order terms of the expansion. Calculation of the exact expansion coefficients at each order by this relation, unlike for the low-ordered terms, could be challenging at times. Therefore, to describe the $n \rightarrow \infty$ behavior of the high-order terms as well as the size of the approximation by seeking an asymptotic expansion of the solution in terms of the pre-factors, we may employ the factorial/power formula as it generates the form of the expansion coefficients, without loss of generality. It is worth to point out that as one may notice these approximated solutions will clearly be not exact when $\varepsilon$ is small but nonzero, they only define their asymptotic equality for sufficiently large $n$ and small $\varepsilon$. Moreover, the presence of the singularity or singularities of (2.7) forces the asymptotic expansion to diverge in the standard factorial divided by a power nature in the limits $n \rightarrow \infty$ and $\varepsilon \rightarrow 0$ as the calculation of the general terms requires the differentiation of the preceding terms at each order.

\section{Asymptotic formula of the high-order terms}

As discussed earlier, finding the exact solution of such equations in the form of (2.1) could be extremely difficult sometimes in the asymptotic procedure. However, as before, our motivation is to study the general asymptotic form of the coefficients for sufficiently large values of $n$ in terms of the pre-factor functions of the particular ODE. These coefficients are governed by the nearest singularity of the expansions. For this reason, we will approximate the higher-order coefficients of the expansion using the powerful factorial/power method as they are naturally divergent in this nature in many cases in the limit $\varepsilon \rightarrow 0$. We consider the high-order terms $w_{n}(z)$ of a function $w(z)$, which is asymptotic to a factorially divergent power series [7, 15], diverge in the following form

$$
w_{n}(z)=\frac{\Gamma(2 n+\beta)}{\chi_{1}(z)^{2 n+\beta}}\left(\sum_{k=0}^{\infty} \frac{A_{k}(z)}{(2 n+\beta)^{k}}\right), \text { as } n \rightarrow \infty,
$$

where $\chi_{1}(z)=0$ at the singular point(s) of the leading order term (2.7), $\beta$ is a constant and $\Gamma$ is the gamma function, or factorial function, as described in [22]; it enables to extend the domain of the factorial to complex arguments for negative values of the non-integers, see [23, pg. 149]. We remark that the ansatz given in (3.1) is the only leading order approximation of the expansion (2.5) or (2.2) in most general form. There must be separate factorial/power ansatz for each singular points exist. It indeed extracts the high-order term behavior of the expansion wherein derivation of the behavior of $\chi_{1}(z)$ plays a pivotal role in the asymptotic procedure. To fully determine all components of the high-order terms in (3.1), we substitute the ansatz (3.1) into the relation (2.8). After performing some computations, we find at the leading order for sufficiently large values of $n$ that

$$
\chi_{1}^{\prime}(z)^{2}+e^{-i \theta} g(z)=0
$$

through which we find that

$$
\chi_{1}^{\prime}(z)= \pm \sqrt{-e^{-i \theta} g(z)}
$$


After having the integration of both sides in (3.2), we subsequently derive the denominator $\chi_{1}(z)$ as a function of the pre-factor $g(z)$ such that

$$
\begin{aligned}
\chi_{1}(z) & = \pm \int \sqrt{-e^{-i \theta} g(z)} d z+c_{\chi_{1}} \\
& = \pm \int \sqrt{\exp (i(\pi-\theta)) g(z)} d z+c_{\chi_{1}},
\end{aligned}
$$

in which $c_{\chi_{1}}$ is an integration constant. $\chi_{1}(z)$ requires to satisfy the singularity or singularities of the early term $w_{0}(z)$, which precisely causes the general terms to diverge, by which the integration constant can be derived. The denominator of the high-order coefficients is expressed as a multiplication of the pre-factor $g(z)$ and $e^{i \theta}$ as a result of the choice of re-scaled $\varepsilon_{1}$ in (2.3). Next, we will focus on deriving the general form of the leading $A_{0}(z)$ term in relation to the pre-factors $f(z)$ and $g(z)$ for sufficiently large values of $n$ since it contributes to the expansion before the subsequent $A_{n \geq 1}(z)$ functions in the limit $n \rightarrow \infty$. To be able to do this, we carry on the next order of balancing when the summation index $n$ is sufficiently large. Similarly to previous order of balancing, after doing the required calculations and simplifications, we attain the differential equation of $A_{0}(z)$ as

$$
\chi_{1}^{\prime \prime}(z) A_{0}(z)+2 \chi_{1}^{\prime}(z) A_{0}^{\prime}(z)+f(z) \chi_{1}^{\prime}(z) A_{0}(z)=0 .
$$

Although it looks the same as its corresponding one in [9], $A_{0}(z)$ of (3.1) will be a complex function as well in this case as the denominator function $\chi_{1}(z)$ depends upon $\theta$ given by the relation in (3.3). Particularly for this expression (3.4), after doing the simple separation and then doing the direct integration with respect to $z$, unknown $A_{0}(z)$ may be evaluated in the following form

$$
A_{0}(z)=c_{0} \frac{\exp \left(-\int \frac{f(z) d z}{2}\right)}{\sqrt{\chi_{1}^{\prime}(z)}} .
$$

Back substitution of the relation obtained in (3.2) into (3.5) completely derives $A_{0}(z)$ as

$$
A_{0}(z)=c_{0} \frac{\exp \left(-\int \frac{f(z) d z}{2}\right)}{[ \pm \exp (i(\pi-\theta)) g(z)]^{1 / 4}} .
$$

Note that all of the integration constants obtained so far can be absorbed into a single constant $c_{0}$, without loss of generality. In this conjecture, substituting all the relations derived by now for $\chi_{1}(z)$ and $A_{0}(z)$ in (3.3) and (3.5) into the factorial/power form in (3.1), we may generate the most general form of the high-order coefficients as following

$$
w_{n}(z) \sim c_{0} \frac{\Gamma(2 n+\beta)}{\chi_{1}(z)^{2 n+\beta}}\left(\frac{\exp \left(-\int \frac{f(z) d z}{2}\right)}{[ \pm \exp (i(\pi-\theta)) g(z)]^{1 / 4}}\right), \text { as } n \rightarrow \infty .
$$

Substituting this coefficient (3.6) back into the summation of the singularly perturbed ODE completes the derivation of the general asymptotic representation of the singular ODE in terms of $f(z), g(z)$ and $t(z)$ of (2.4) in powers of $\varepsilon$, wherein $t(z)$ and its zeros are crucial while deriving the low-ordered terms of the expansion, so does the high-order terms. Finally, to establish the most general form of the solutions by the complex values of $\varepsilon_{1}$, we should use the equation (2.3) and leave $\varepsilon$ alone. Once doing this and substituting it into the summation, we establish the leading order approximation of $w(z)$ as a function of pre-factor functions in powers of $\varepsilon_{1}$ and $\exp (-i \theta)$ such that

$$
w(z) \sim c_{0} \sum_{n=0}^{\infty}\left(\varepsilon_{1} e^{-i \theta}\right)^{n} \frac{\Gamma(2 n+\beta)}{\chi_{1}(z)^{2 n+\beta}}\left(\frac{\exp \left(-\int \frac{f(z) d z}{2}\right)}{[ \pm \exp (i(\pi-\theta)) g(z)]^{1 / 4}}\right), \text { as } n \rightarrow \infty,
$$

which is the leading high-order behavior of the asymptotic solution $w(z)$ of equation (2.1) derived based on using the factorial/power representation (3.1) with the limit $n \rightarrow \infty$. The choice of rescaling $\varepsilon_{1}$ permits us to expand (2.1) as a power series of its complex values. Again, the region of its validity depends on its singularity structure which may be addressed via exponential asymptotics and it preserves all the features of the differential equation. Because the exact solutions of such type of equations are rare in physics applications, one can implement this for the suitable choices of the pre-factor functions and can find the limiting behavior of $w(z)$ when needed. However, if it is not sufficient, this means that the perturbation parameter is not small enough. Furthermore, as this expansion is naturally divergent due to increasing powers of the low-ordered terms, by taking the ratio of the adjacent terms of the expansion, a general form of the optimal truncation point as well as the relation of the resultant remainder, which is exponentially small, and divergent series can be directly and easily formulated and interpreted by these specified formulas. 


\section{Concluding remarks}

This work has taken into consideration how to straightforwardly address the general form of the tail of the expansions for complex $\varepsilon_{1}$ by focusing on the pre-factor functions of the certain ODEs in the form of this paper along with their effects in the asymptotic expansions. The obtained links in relation to pre-factors can be implemented for the complex values of $\varepsilon_{1}$, whence they are extendable to complex region. Moreover, as one may notice that being $\varepsilon_{1}$ complex turns the pre-factors and the right-hand side of the inhomogeneous singular equations into complex factors. Therefore, the formulas we have attained are applicable for the study of the singular ODEs having the complex pre-factors as well.

Competing interest: The author declares that no competing interests exist.

\section{Acknowledgement}

I would like to thank the referees for their helpful comments on the manuscript.

\section{References}

[1] H. Poincaré, Sur les intégrales irrégulières, Acta Math., 8 (1) (1886), 295-344.

[2] E. J. Hinch, Perturbation Methods, Cambridge University Press, Cambridge, 1991

[3] M. Van Dyke, Perturbation Methods in Fluid Mechanics, Academic Press, New York, 1964.

[4] C. M. Bender, S. A. Orszag, Advanced Mathematical Methods for Scientists and Engineers I: Asymptotic Methods and Perturbation Theory, Reprint of the 1978 original, Springer-Verlag, New York, 1999

[5] V. F. Zaitsev, A. D. Polyanin, Handbook of Exact Solutions for Ordinary Differential Equations, 2nd edition, Chapman \& Hall/CRC Press Company, Boca Raton, FL, 2003.

[6] J. Cousteix, J. Mauss, Successive Complementary Expansion Method, Asymptotic Analysis and Boundary Layers, Springer, 2007, pp. 59-98.

[7] R. B. Dingle, Asymptotic Expansions: Their Derivation and Interpretation, Academic Press, London-New York, 1973.

[8] M. V. Berry, Asymptotics, superasymptotics, hyperasymptotics, H. Segur, S. Tanveer, H. Levine (editors), Asymptotics Beyond All Orders, NATO Adv. Sci. Inst. Ser. B Phys., vol. 284, Springer Science \& Business Media, Boston, 1991, 1-14

[9] F. Say, Late-order terms of second order ODEs in terms of pre-factors, Hacet. J. Math. Stat., (in press).

[10] M. V. Berry, Uniform asymptotic smoothing of Stokes's discontinuities, P. Roy. Soc. Lond. A Mat., 422 (1862) (1989), 7-21.

[11] M. V. Berry, Stokes's phenomenon; smoothing a Victorian discontinuity, Inst. Hautes Études Sci. Publ. Math., 68 (1) (1988), 211-221.

[12] B. L. J. Braaksma, G. K. Immink, M. Van der Put, J. Top (editors), Differential equations and the Stokes phenomenon, Proceedings of the workshop held at the University of Groningen, Groningen, May 28-30, 2001, World Scientific Publishing Co., Inc., River Edge, NJ, 2002, doi:10.1142/5107.

[13] P. J. Langman, When is a Stokes line not a Stokes line?, Ph.D. Thesis, University of Southampton, 2005.

[14] M. D. Kruskal, H. Segur, Asymptotics beyond all orders in a model of crystal growth, Stud. Appl. Math., 85 (2) (1991), 129-181.

[15] J. P. Boyd, The devil's invention: Asymptotic, superasymptotic and hyperasymptotic series, Acta Appl. Math., 56 (1) (1999), 1-98.

[16] F. W. J. Olver, Asymptotic expansions of the coefficients in asymptotic series solutions of linear differential equations, Methods Appl. Anal., 1 (1) (1994), 1-13.

[17] R. E. Meyer, A simple explanation of the Stokes phenomenon, SIAM Rev., 31 (3) (1989), 435-445.

[18] J. P. Boyd, A hyperasymptotic perturbative method for computing the radiation coefficient for weakly nonlocal solitary waves, J. Comput. Phys., 120 (1) (1995), 15-32.

[19] F. Say, Exponential asymptotics: multi-level asymptotics of model problems, Ph.D. Thesis, University of Nottingham, 2016.

[20] R. E. Meyer, Exponential asymptotics, SIAM Rev., 22 (2) (1980), 213-224.

[21] P. Hsieh, Y. Sibuya, On the asymptotic integration of second order linear ordinary differential equations with polynomial coefficients, J. Math. Anal. Appl., 16 (1) (1966), 84-103.

[22] M. Abramowitz, I. A. Stegun, Handbook of Mathematical Functions: With Formulas, Graphs, and Mathematical Tables, Reprint of the 1972 edition, Dover Publications, Inc., New York, 1992.

[23] E. C. Titchmarsh, The Theory of Functions, Oxford University Press, Oxford, 1939. 